\title{
EL GESTO "AGITAR LA MANO": UN INSUMO \\ PARA LA ENSEÑANZA DE ELE EN EL CONTEXTO \\ COSTARRICENSE
}

Silvia Rivera Alfaro

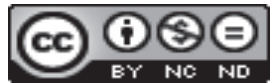

Esta obra está bajo una licencia Creative Commons

Reconocimiento-No Comercial-Sin Obra Derivada 



\title{
EL GESTO "AGITAR LA MANO": UN INSUMO PARA LA ENSEÑANZA DE ELE EN EL CONTEXTO COSTARRICENSE
}

\author{
THE “SHAKING THE HAND” GESTURE: AN INPUT FOR THE \\ TEACHING OF ELE IN THE COSTA RICAN CONTEXT
}

\author{
Silvia Rivera Alfaro
}

\begin{abstract}
RESUMEN
Este trabajo describe un gesto a partir del conocimiento pragmático de hablantes nativos con el fin de comprender sus usos en la comunicación cara a cara en el habla costarricense. Para el análisis se utiliza la clasificación de gestos de Knapp (1982). Metodológicamente, se recogió información de fuente primaria por medio de un cuestionario semiabierto disponible en línea. Entre los resultados destaca que el gesto se usa en distintos contextos, y aparece en varias categorías con diversas funciones. El estudio busca aportar al conocimiento de elementos no verbales para la clase de ELE en el contexto costarricense.

Palabras clave: ELE, gestos, comunicación no verbal, español, Costa Rica.
\end{abstract}

\begin{abstract}
This paper describes a gesture from the native speakers' pragmatic knowledge with the aim of understanding its uses in face to face communication in Costa Rican speech. The analysis is done using the classification of gestures exposed by Knapp (1982); methodologically, the speakers' information was taken using a semi open online questionnaire. Among the results, it should be pointed out that the studied gesture is used in different contexts, and appears in different categories with diverse functions. The study contributes to the knowledge of non-verbal elements for the class of Spanish as a foreign language (ELE) in the Costa Rican Context.
\end{abstract}

Key words: ELE, gestures, non-verbal communication, Spanish, Costa Rica.

\section{Introducción}

La comunicación cara a cara presenta un carácter multimodal (Payrató, 2006); es decir, que no se lleva a cabo únicamente por medio de lo verbal, sino que en ella aparecen distintos modos (lenguajes) que permiten construir el sentido. Por esta razón, es bien sabido que para permitirles a nuestros alumnos y alumnas de español como lengua extranjera (ELE)

\footnotetext{
Bach. Silvia Rivera Alfaro. Universidad de Costa Rica. Docente, Programa de Español como Lengua Extranjera. Correctora de estilo, Revistas e-Ciencias de la Información y Actualidades Investigativas en Educación. Costa Rica. Correo electrónico: silvia.riveralfaro@gmail.com
}

Recepción: 15- 05- 2015

Aceptación: 26- 10- 2015 
llegar a comunicarse en la lengua meta es necesario abordar en clase no solo lo verbal, sino también los aspectos socioculturales y el lenguaje gestual (por ejemplo: Yagüe-Barredo, 2004; Betti y Costa, 2006; Gaviño-Rodríguez, 2012; Martinell-Gifre y Ueda, s.f.; Ciarra-Tejada, 2011; Monterrubbianesi, 2013; Sánchez-Benítez, 2009). A pesar de esto, en Costa Rica son prácticamente nulos los trabajos que abordan aspectos no verbales y pragmáticos; básicamente, se encuentra un estudio sobre la risa (Bolaños-Carpio, 2009). En otras palabras, en el país la persona docente de ELE no cuenta con los insumos necesarios para abordar este tema en el aula.

Tomando en cuenta lo anterior, este trabajo surge de las interrogantes de un grupo de nivel intermedio del Programa de Español para Extranjeros (PEE) de la Universidad de Costa Rica (UCR). Las estudiantes y los estudiantes preguntaron por un gesto que algunas personas de las familias que les brindaban hospedaje realizaban con mucha frecuencia al entablar una conversación con ellas y ellos. Específicamente, expresaron a su docente la duda sobre un gesto que consiste en mover o agitar la mano repetidamente. Para el estudiantado del curso resultaba particularmente interesante que el gesto presentara un sonido; además, existía una preocupación por la rapidez con la que se llevaba a cabo y porque les resultaba inentendible. De hecho, alguna persona extrajera manifestó que pensaba que "algo le sucede en la muñeca a la persona"; es decir, no era identificado como un gesto que buscaba comunicar sino como una condición o algo que le sucedía a quien lo utilizaba.

Ante las consultas del grupo, este estudio experimental tuvo por objetivo describir, a partir del conocimiento pragmático de los/as hablantes, un gesto utilizado comúnmente en Costa Rica para la comunicación cara a cara con el fin de categorizar y comprender la función de este comportamiento no verbal cinésico desde un enfoque pragmático. Específicamente, el trabajo buscó: establecer los contextos y las situaciones en las cuales se utiliza este gesto; clasificarlo de acuerdo con las características descritas por Knapp (1982) para el movimiento cinésico; y definir algunas de sus funciones en la comunicación. La finalidad de llevar a cabo la pesquisa fue generar los datos necesarios que puedan servir como insumo para abordar este gesto en la clase de ELE.

\section{El gesto estudiado}

Como punto de partida, se presenta una descripción detallada del gesto, la posición de la mano y se abordan otros gestos similares que han sido estudiados. Para la descripción de la secuencia de movimientos se utiliza el concepto de frase gestual de Poyatos (2002); la frase incluye tres momentos para la realización de cualquier movimiento: fase de formación, fase central y fase de cierre.

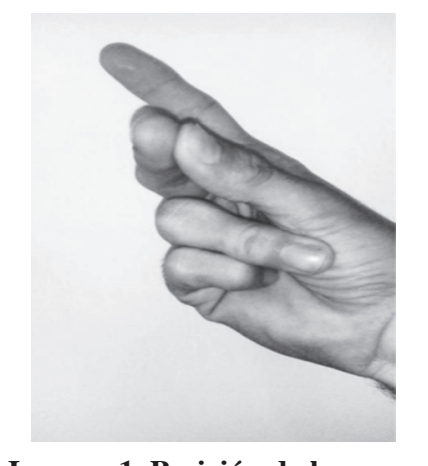

Imagen 1. Posición de la mano

Fuente: Elaboración propia con la colaboración de Jorge Mora Murillo 
Como se mencionó el gesto se realiza moviendo o agitando la mano de arriba a abajo mientras se realiza un sonido que es causado por el mismo movimiento. En la Imagen 1 se puede apreciar la posición que toma la mano para llevar a cabo el gesto. La yema del dedo del corazón se toca con la del pulgar, mientras el anular y el meñique tocan la palma de la mano. Por su parte, el dedo índice está libre y relajado; por esta razón, golpea el dedo del corazón cuando se ejecuta el movimiento rápido.

En la Imagen 2 se ilustra el movimiento que se realiza para este gesto. La secuencia de imágenes debe leerse de izquierda a derecha y por línea, como se hace con las tiras cómicas. En la imagen se puede observar la frase gestual de este gesto. Primero, en la fase de formación, la mano toma la posición mostrada en la Imagen 1 y el brazo asciende desde abajo (no siempre lo hace hasta el hombro, como lo realiza la persona de la imagen). Luego, en la fase central la mano baja y sube repetidas veces con fuerza y velocidad en un ángulo de aproximadamente $45^{\circ}$ o en una posición paralela al cuerpo; para esta fase en la figura se ilustra únicamente un movimiento que baja y sube dos veces, pero en el habla cotidiana el movimiento puede repetirse un número de veces mayor. Finalmente, en la fase de cierre la mano vuelve a la posición inicial.
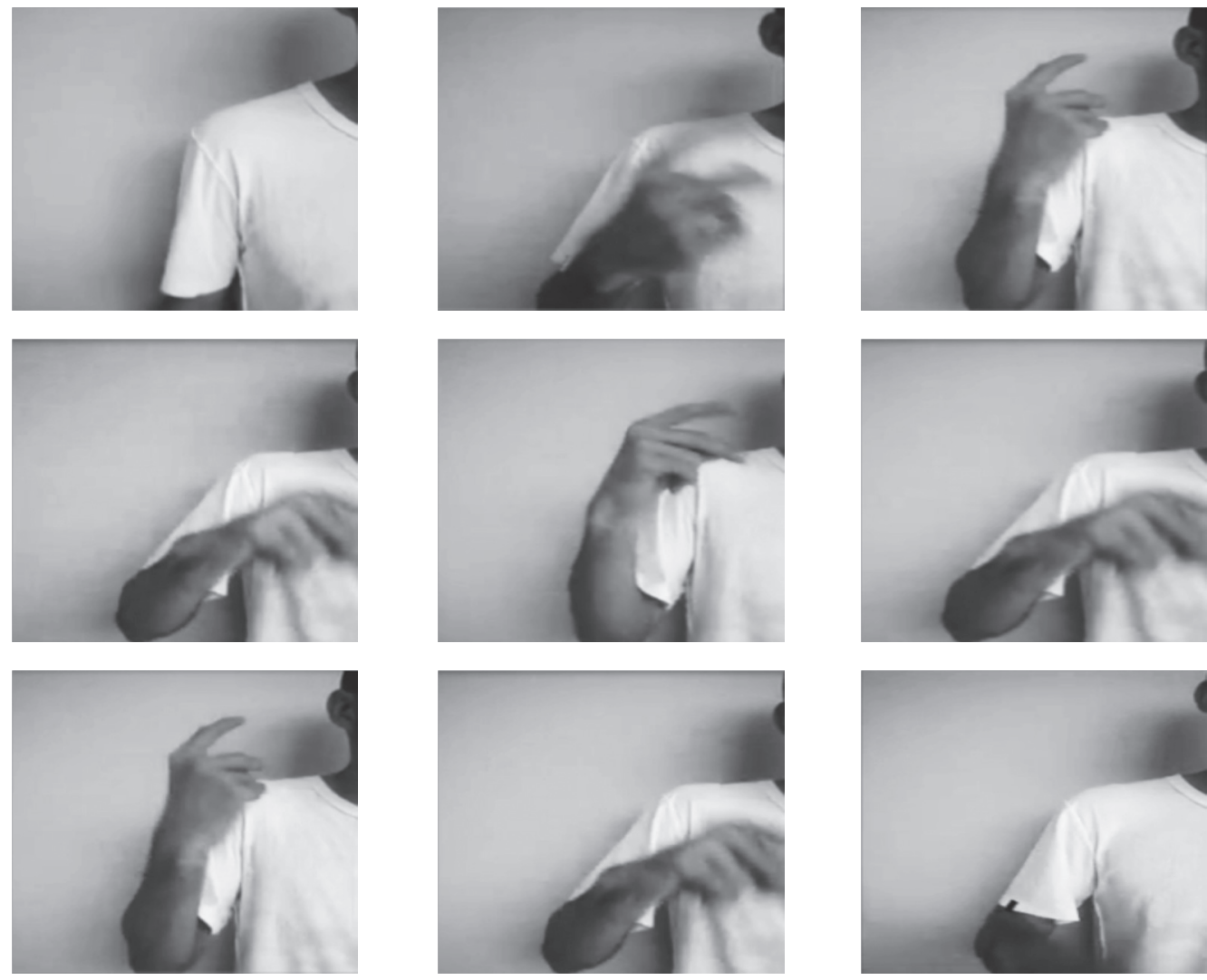

Imagen 2. Movimiento del gesto

Fuente: Elaboración propia con la colaboración de Jorge Mora Murillo

Existe la posibilidad de que este mismo gesto se realice utilizando la mano y la cara al mismo tiempo. En la Imagen 3 se muestra esta realización. La frase gestual de la mano es la misma. La boca se mantiene en la misma posición durante toda la fase central del movimiento. 


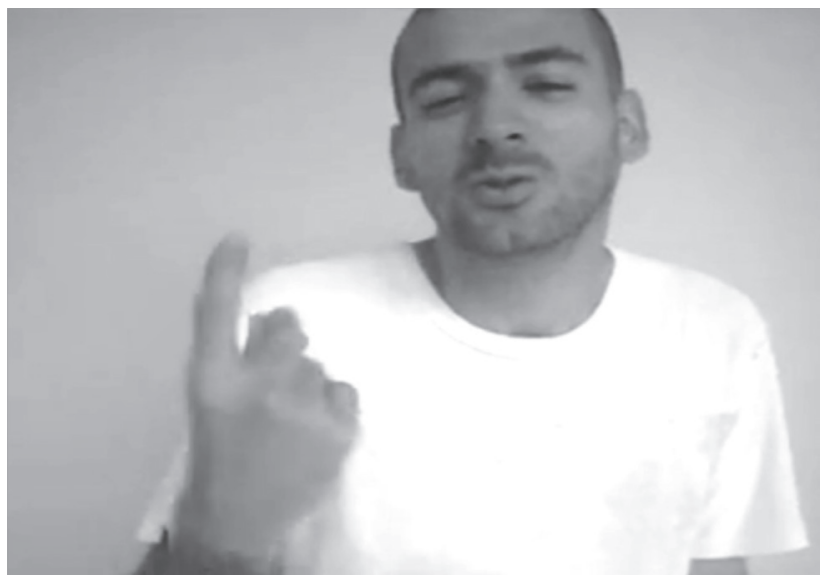

Imagen 3. Gesto con mano y expresión facial

Fuente: Elaboración propia con la colaboración de Jorge Mora Murillo

Esta variedad del gesto (con la posición de la mano de la Imagen 1) fue la única que incluyeron las personas que cursaban el nivel intermedio y consultaron sobre este tema. No obstante, para este gesto podría existir otra realización con la mano extendida y sin ningún sonido, pues previo a la elaboración del cuestionario que se utilizó para la investigación se realizó una consulta exploratoria a varias personas hablantes del español de Costa Rica y algunas expresaron que sí reconocían el gesto descrito por el alumnado, pero lo ejecutaban de ese otro modo. Es posible que esta otra realización del movimiento no resultara igual de llamativa para los estudiantes y las estudiantes de español o que la consideraran como otro gesto distinto por su poca familiaridad con las señas del país.

De esta última variedad con la mano extendida, se encuentra un movimiento similar reportado para el español peninsular en el diccionario de gestos de Martinell-Gifre y Ueda (s.f.), que está basado en otros diccionarios a los que no se ha podido tener acceso, pero que son todos sobre la misma variedad del español. Este mismo movimiento aparece mencionado en uno de los archivos didácticos de Video ELE (Iruela, s.f.), tal como se muestra en la Imagen 4. Mientras en el diccionario para el gesto se utiliza únicamente la mano, en el video didáctico el gesto incluye el movimiento de la mano unido a una expresión facial. Esta diferencia podría deberse al hecho de que en el diccionario se representa una conversación y en el video de Iruela el gesto se realiza en silencio mientras una voz en off enuncia el sentido que se le atribuye al movimiento.

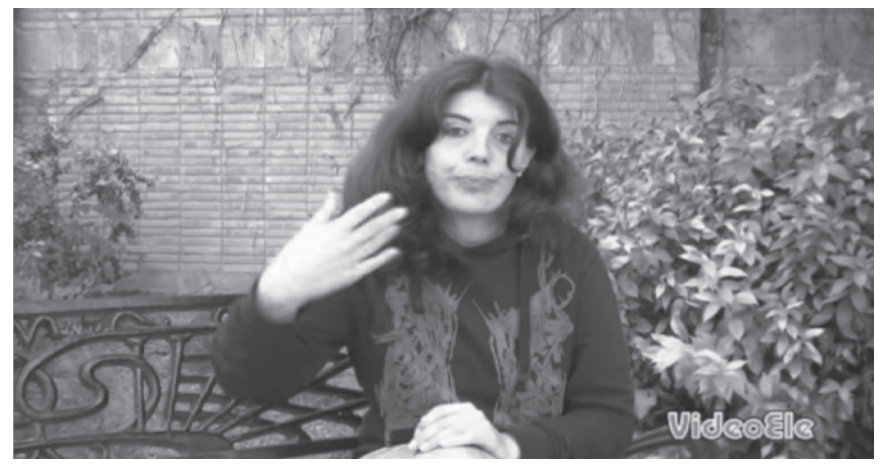

Imagen 4. Gesto descrito para el español peninsular

Fuente: Iruela (s.f., min 1:15') 
En ambos documentos se le atribuye un único significado al gesto: “mucho"; este será un aspecto al que volveremos adelante. La frase gestual es la misma que la del gesto utilizado en Costa Rica: en la fase de formación la mano sube y se va colocando en la posición que requiere, en la fase central se realizan movimientos repetidos en un ángulo similar y en la fase de cierre se vuelve a la posición inicial.

Resulta necesario mencionar que el gesto peninsular no está reportado en el diccionario de Gaviño-Rodríguez (2012). Tampoco se tuvo acceso a ningún trabajo que mencionara este o algún gesto similar, como el peninsular, para otras variedades de español de América, aunque es posible que sea utilizado en algunos países.

\section{Marco teórico}

En este trabajo se toma en cuenta el concepto de comunicación tal como lo presenta Bravo (2012): “...una actividad humana inserta en otras actividades humanas, donde los actos que se realizan son comunicativos (...) se utilizan recursos no sólo verbales, sino también no verbales" (p. 104). Al analizar un gesto, el estudio se centra en un recurso no verbal específico. Se afirma que se analiza desde el conocimiento pragmático de las personas hablantes de esta variedad del español, pues -como se verá en la metodología- se pregunta a las informantes y los informantes por el uso lingüístico contextualizado.

Para el análisis del recurso seleccionado, se utiliza la recopilación teórica que presenta Knapp (1982) sobre la comunicación no verbal; específicamente, se toman en cuenta especialmente las secciones relacionadas con el movimiento. En su trabajo, el autor problematiza la noción de comunicación no verbal (también lo hacen autores y autoras que analizan los aspectos multimodales), pues tanto el término no verbal como el de comunicación presentan diversas posibles interpretaciones (1982, p. 15) y es difícil comprender cuál es el límite entre lo verbal y lo no verbal, o si lo no verbal es la señal que se emite o la interpretación que se le asigna verbalmente (1982, p. 16). En esta dirección, debe señalarse que al analizar un gesto (o cualquier elemento no verbal) lo convertimos en lenguaje verbal y que esta es una limitación metodológica que aparece básicamente en todos los trabajos de este tipo.

Se sigue a Knapp y otros autores para afirmar que no es posible separar lo verbal de lo no verbal en la comunicación o interacción cara a cara (1982, p. 15 y p. 32): desde la perspectiva expuesta por Knapp, tanto lo verbal como lo no verbal son comportamientos o conductas que están íntimamente relacionados. De las diversas conductas no verbales, este trabajo se enfoca el movimiento del cuerpo o comportamiento kinésico, que es

\footnotetext{
los gestos, los movimientos corporales, los de las extremidades, las manos, la cabeza, los pies y las piernas, las expresiones faciales (sonrisas), la conducta de los ojos (parpadeo, dirección y duración de la mirada y dilatación de la pupila), y también la postura (Knapp, 1982, p. 17)
}

Knapp presenta una clasificación para estas conductas; los movimientos corporales son: emblemas, ilustradores, muestras de afecto, reguladores o adaptadores. De todos los anteriores, los emblemas -como se verá a continuación- son el único tipo que presenta un significado preciso, pues los demás no presentan uno (Knapp, 1982, p. 179). Dicho esto se explicará cada una de las categorías.

El primer tipo son los emblemas. Se caracterizan por poder "traducirse" a una o dos palabras o una frase (Knapp, 1982, p.17), con el requisito de que dicha traducción debe ser conocida por la mayoría de los miembros de un grupo de comunicación (Knapp, 1982, p. 180); es decir, se requiere que exista un acuerdo entre los hablantes con respecto a su significado. 
Según Knapp (1982), los emblemas se caracterizan por: ser específicos de cada cultura, aunque algunos se repitan o asemejen en más de una cultura, pues transmiten mensajes semejantes (p. 18); producirse generalmente con las manos, aunque también con la cara (p. 18); utilizarse para comunicar cuando el canal verbal está bloqueado (pp. 18-19); interpretarse a partir del contexto tal como sucede con los intercambios verbales (p. 19); no formar series de palabras (con excepciones) (p. 20) y existir algunos específicos de subgrupos (p. 20), como los que podrían crearse dentro de una cultura juvenil.

Los ilustradores, que son el segundo grupo, son actos que están muy relacionados con el discurso oral (Knapp, 1982, p. 180), pues aparecen acompañándolo. Estos actos

\begin{abstract}
sirven para ilustrar lo que se dice verbalmente. Pueden ser movimientos que acentúen o enfaticen una palabra o frase, esbocen una vía de pensamiento, señalen objetos presentes, describan una relación espacial o el ritmo de un acontecimiento, tracen un cuadro del referente o presenten una acción corporal. También puede haber emblemas que se utilicen para ilustrar juicios verbales, bien repitiendo o bien sustituyendo una palabra o una frase (Knapp, 1982, p. 20)
\end{abstract}

Knapp compara los ilustradores con los emblemas. Según el autor, la diferencia entre ellos radica en que los emblemas se utilizan de manera más "deliberados y explícitamente consciente", mientras que los ilustradores no son realizados de manera tan claramente consciente como los emblemas, aunque se utilizan intencionalmente para comunicar y son parte del campo consciente también (Knapp, 1982, p. 20). Para él, los ilustradores se muestran con más frecuencia en situaciones comunicativas donde media algún sentimiento, por ejemplo, "personas excitadas o entusiasmadas (...) e igualmente son de prever más ilustradores en situaciones «difíciles» de comunicación” (Knapp, 1982, p. 21). En otras palabras, puede afirmarse que se utilizan cuando una persona no logra explicarse o si los interlocutores no se entienden bien. Para el autor, existe la posibilidad de que los ilustradores sean aprendidos por medio de la observación de las demás personas.

El tercer tipo son las muestras de afecto. Se trata de expresiones de estados afectivos que se manifiestan por medio de la cara como fuente primaria del afecto, aunque también por medio del cuerpo (puede comprenderse como juicios globales sobre afectos). Según Knapp, estos comportamientos no buscan comunicar, pero pueden ser intencionales (1982, p. 21). En este aspecto, este trabajo difiere de la propuesta de Knapp, pues no se considera la existencia de elementos que no comuniquen. La diferencia surge al comprender la lengua como un instrumento social con el que se llevan a cabo actos y que presenta distintas funciones, lo cual se puede relacionar directamente con la perspectiva pragmática, por ejemplo, con las actividades de negociación de imagen (Bravo, 2012).

Los reguladores son el cuarto tipo de actos no verbales. Mantienen y regulan el discurso en la interrelación entre sujetos: "Indican al hablante que continúe, repita, se extienda en detalles, se apresure, haga más ameno su discurso, conceda al interlocutor su turno al hablar, y así sucesivamente" (Knapp, 1982, p. 21). Estas señales se caracterizan por llevarse a cabo de manera casi inconsciente e involuntaria, aunque son fáciles de identificar conscientemente al verlas en los otros (Knapp, 1982, p. 22), pues las personas están acostumbradas a prestar atención a dichas señales en la comunicación cotidiana. En la conversación los reguladores sirven para: iniciar una conversación (saludo), finalizar un intercambio (despedida o señales físicas que indican que se desea acabar la conversación), mantener la conversación (por ejemplo, funcionando como retrocanalizadores), anunciarle al interlocutor o interlocutores que es un lugar apropiado para el cambio de turno (LAT) o que se está renunciando al turno (Knapp, 1982, pp. 187-192). 
El último grupo son los adaptadores. Para Knapp estos no tienen una finalidad comunicativa, aunque pueden aparecer acompañando la comunicación verbal en ciertas situaciones (1982, p. 23); son “...adaptaciones conductuales que ejecutamos respondiendo a ciertas situaciones de aprendizaje” (p. 192); y, se subdividen en tres tipos según hacia quién estén dirigidos: audoadaptadores, heteroadaptadores y adaptadores dirigidos a objetos (pp. 22-23). En este trabajo también se cuestiona este grupo a partir de la definición de lenguaje que se adopta y se considera que, si bien una conducta puede emitirse "sin una finalidad comunicativa", la persona receptora puede interpretarla y, por ello, encontrar en el movimiento un significado que no depende de las intenciones de quien lo lleva a cabo.

Adicional a la clasificación recién expuesta, en este trabajo se toma en cuenta las funciones del comportamiento no verbal. Knapp describe seis funciones que no son específicas para el movimiento cinésico, sino que aplican para el comportamiento no verbal en general. La primera es la repetición de lo que se indica por medio de las palabras (Knapp, 1982, p. 27). La segunda es la contradicción de lo dicho verbalmente (Knapp, 1982, p. 27). La tercera es la sustitución de los mensajes verbales (Knapp, 1982, p. 29). La complementariedad, que es la cuarta, "puede modificar o elaborar mensajes verbales (...) sirven como señal de las actitudes e intenciones de una persona respecto a la otra" (Knapp, 1982, p. 30). La quinta es la acentuación o énfasis de partes del mensaje; con respecto a esta el autor señala que "A menudo los movimientos de cabeza o de manos se usan para acentuar el mensaje verbal” (Knapp, 1982, p. 30). La última es la regulación de la conversación; por ejemplo, un elemento no verbal puede guiar los cambios de turno, como retrocanalizadores (Knapp, 1982, pp. 30-31).

\section{Metodología}

Este trabajo es un estudio exploratorio de tipo cualitativo. Para describir el gesto a partir del conocimiento pragmático de las personas hablantes del español de Costa Rica, se recogió una muestra que permitiera hacer referencia a las categorías propuestas por Knapp.

La muestra fue recogida de fuente primaria por medio de un cuestionario semiabierto. El cuestionario incluyó dos videos con el ademán específico del estudio y una serie de preguntas cerradas y abiertas que habían sido establecidas a partir de la propuesta teórica de Knapp. El primer video -del cual proviene la Imagen 2- incluyó el gesto realizado únicamente con la mano para saber si las personas lo reconocían y presentaba sentido; si las personas contestaban afirmativamente en esta parte del cuestionario, se podía considerar que el gesto es manual. Si las personas informantes no reconocían el gesto como únicamente manual, cabía la posibilidad de que lo hicieran con mano y cara, por eso el segundo video - del cual proviene la imagen 3- incluyó esta combinación para ver si la cara hacía parte del gesto.

El cuestionario se publicó en línea y se pidió a las personas informantes que invitaran a otras a contestar con el fin de recolectar una muestra pequeña. De este modo se trabajó con la opinión de un grupo de once informantes compuesto por cinco hombres y seis mujeres. El sexto cuestionario completado por una mujer se utilizó para reemplazar uno de otra que indicó no reconocer el ademán.

El dato de dicha informante resulta de interés, pues para el trabajo exploratorio se tomó en cuenta como única variable el sexo. Claramente, como se trabaja con una muestra no representativa por el limitado número de informantes, se sabe que es imposible realizar afirmaciones que busquen dar respuestas definitivas sobre el gesto tomando en cuenta esta 
variable; no obstante, se consideró útil explorar su uso. En otras palabras, la variable se incluye únicamente para vislumbrar si parece existir una diferencia en la utilización del gesto entre hombres y mujeres que pudiera ser estudiada en trabajos más extensos. Además del dato sobre el sexo de las personas participantes, cabe indicar que todas las personas que colaboraron eran menores de 30 años, aunque no se había establecido previamente un grupo etario en el que se enfocara el estudio.

Las respuestas de las personas hablantes se analizaron de acuerdo con la propuesta de Knapp. Primero, se caracterizó el gesto de acuerdo a la tipología propuesta por el autor: emblemas, ilustradores, muestras de afecto, reguladores y adaptadores. Luego, a partir de las respuestas obtenidas en el cuestionario, se clasificaron sus funciones: repetición, contradicción, sustitución, complementariedad, acentuación, regulación.

\section{Desarrollo}

En esta sección, primero se hace una breve descripción del gesto a partir de la información brindada por las personas informantes. En seguida, se presenta el significado que establecen las personas informantes a partir de los videos. Después, se clasifica el movimiento de acuerdo a la propuesta de Knapp y se establece su función. Finalmente, se identifican contextos en los cuales se utiliza este ademán según el conocimiento de quienes participaron completando el formulario.

\subsection{Descripción y definición del gesto}

Al observar el primer video, de los diez informantes solo tres consideran que el gesto se realiza únicamente con la mano. Las otras siete personas indicaron que el gesto requiere otra parte del cuerpo, no sólo el movimiento de la mano. De estas siete, cuatro mencionaron que se acompaña la mano con la boca, dos incluyeron los ojos y uno de los que indicó los ojos mencionó también la cara. A estas partes del cuerpo, dos informantes mujeres agregan otros elementos de comunicación no verbal: una indicó que tono de la voz (con la cara) tiene un sentido de advertencia y la otra escribió que se realiza un sonido cuando se hace el gesto, en lo cual concuerda con la descripción del estudiantado de ELE.

Al pedir una primera descripción del movimiento o su significado, con el objetivo de que este concepto funcionara como punto de partida sin que el cuestionario pudiera afectar la idea que las personas tuvieran sobre el gesto, cuatro lo relacionaron directamente con una frase específica: “QQué torta!”; seis lo asocian con apuro, preocupación o transgresión de una norma, es decir, que hablan de la emoción del hablante acerca de lo que está diciendo; y uno lo asoció con una identidad cultural específica: "reguetonero como de afirmación". Esa primera definición fue dada por las informantes a partir del video que solo mostraba la mano; al final del cuestionario se mostró el video que permitía además ver la expresión de la cara. Con las respuestas dadas para el significado del gesto tras este último video se evidenció que junto con una expresión facial puede tener muchos significados adicionales: "iqué torta!", "ipóngale!”, “que están hablando de un problema muy grande, o que está viendo a una muchacha muy guapa”, "UUUUYY! Alerta algo de agobio, preocupación y picardía”. Al final del cuestionario, al ver la expresión facial dos de las personas informantes comentaron que la expresión cambiaba el sentido que ellos inicialmente pensaron para el ademán. 


\subsection{Contextos de uso}

A pesar de que las diez personas conocían el gesto, solo siete lo utilizan. Las tres personas que no lo utilizan son mujeres, igual que la persona del onceavo cuestionario que no reconoció el gesto. Este es el único aspecto en el cual la variable sexo resultó pertinente. A las siete personas -mayoritariamente hombres- que sí utilizan el gesto se les solicitó información sobre el contexto.

Primero, se preguntó por las personas con las cuales se utilizaría el gesto para comprender con qué tipo de participantes de una situación comunicativa se elegiría este ademán. De las siete personas cuatro indicaron que lo utilizan en ámbitos de cercanía y solidaridad (amigos, familiares, compañeros, personas de confianza), dos indicaron que lo utilizarían con cualquier persona y un informante indicó que lo utilizaría con "alguien que le pasó algo malo". Una persona aclaró que no lo usaría con profesores o funcionarios de alguna institución por parecerle muy informal.

Luego, se preguntó por lugares donde se realiza este gesto, para saber si existen espacios que los usuarios consideren como inapropiados para su utilización. Al respecto, cuatro indicaron que lo realizarían en cualquier sitio; dos no indican un lugar específico, sino que hacen referencia a situaciones (“una situación fea”, "en una reunión de amigos...”); y una persona señaló lugares específicos en los que sí lo usaría (la calle, el aula y la casa) y estableció como espacios en donde no lo realizaría (el banco ni en una oficina), a estos añadió una situación (una conferencia). Se repitió en esta pregunta el señalamiento de este movimiento como informal, dos personas indicaron que no lo utilizarían en un ambiente formal. La persona que había establecido lugares específicos donde no realizaría el gesto, justificó su respuesta afirmando que "sería poco serio hacer el gesto" en esos lugares.

Posteriormente, para describir mejor el contexto en que se realiza el gesto, se solicitó a sus usuarios describir una situación típica (o varias, según prefirieran) en la que utilizarían el gesto. El tipo de situaciones que indican incluyen -en sus propias palabras-: una conversación con compañeros sobre un tema que impacta, encontrar un recibo con un precio elevado (impactante), luego de cometer un error (sabiendo que se recibirá un regaño), luego de que alguien le cuente un problema o que cometió un error, luego de que alguien comete un error (para indicarle que se sabe que está en problemas) y como respuesta a algo que la otra persona está diciendo y le causa impacto. Como se evidencia en estas descripciones, quienes utilizan el gesto lo hacen en gran parte como respuesta a otra persona, es decir, para mantener la interacción o facilitarla.

\subsection{Clasificación del gesto}

Para llevar a cabo la clasificación del movimiento cinésico se realizó una serie de preguntas relacionadas con cada una de las categorías; algunas de ellas relacionaban las categorías entre sí, especialmente cuando se trataba de categorías que pudieran confundirse.

Primero, se preguntó si el gesto podría ser traducido a palabras, para saber si este gesto funciona como emblema. Al respecto, la mitad de las personas informantes piensa que sí se puede traducir (dos mujeres y tres hombres); las otras cinco opinan que no es posible (tres mujeres y dos hombres). Entre quienes atribuyeron un significado al gesto, cuatro lo tradujeron como "iqué torta!" y uno escribió "reacción ante situación límite”. Esta última frase se toma como una explicación y no como una traducción, pues -como se vio a partir de Knapp- esta 
tendría que ser una frase o un par de palabras que funcionen como definición de diccionario. Además, siguiendo al teórico, los emblemas se caracterizan por tener un significado que es compartido por la comunidad y por estar dentro del campo consciente; por eso, este primer desacuerdo no permite incluirlo en esta clasificación por completo, aunque sí es posible que para algunos grupos funcione como emblema.

Tomando en cuenta que tanto los emblemas como los ilustradores entran dentro de los movimientos más conscientes de los hablantes y que los emblemas tienen significado por sí mismos, se preguntó si este gesto aparece acompañado por palabras siempre, si no las requiere o si puede aparecer de cualquiera de las dos maneras anteriores. A esta pregunta, dos informantes respondieron que se puede utilizar en silencio; sin embargo, estas personas habían indicado que no se podría traducir a una frase, por lo cual sus respuestas no lo categorizarían como emblema. Las otras ocho personas respondieron que podría usarse acompañando el habla o utilizarse en silencio; es decir, las cinco personas que lo catalogaron como emblema, mantuvieron abierta dicha posibilidad. A esto se suma el hecho de que existen emblemas que pueden usarse para ilustrar juicios verbales por medio de la sustitución o repetición de una palabra; no obstante, el desacuerdo entre la posibilidad de traducir el movimiento o no sigue siendo un argumento en contra para categorizarlo como emblema.

La opinión de las personas usuarias descrita hasta aquí también abre la posibilidad de que el gesto funcione como ilustrador, pues los ilustradores acompañan al discurso oral. Especialmente, debe conferirse atención al hecho de que los ilustradores pueden acentuar o enfatizar lo que se está diciendo, porque al preguntar (por medio de selección múltiple) para qué sirve este gesto, siete personas eligieron enfatizar o intensificar lo que se está diciendo. También, una persona seleccionó que permite aclarar lo que se está diciendo, otra indicó que permite alarmar y una dijo que sirve para marcar un error, siendo todas estas funciones de los ilustradores.

En esa misma pregunta se buscó definir si este movimiento podría funcionar como regulador preguntando si servía para iniciar o finalizar una la conversación o para mostrar que la otra persona podría hablar (LAD): ninguna de estas opciones fue seleccionada. No obstante, este ademán puede funcionar como retrocanalizador, pues en general se encontró que puede utilizarse para demostrar al otro que se sigue la historia que está diciendo y dar muestras de afecto; precisamente, el afecto es la siguiente categoría propuesta por Knapp.

La categoría de muestras de afecto genera especiales problemas para su análisis, pues como se señaló en el marco teórico según Knapp no buscan comunicar, pero sí son intencionales. Para establecer si este gesto puede tener esta función, se preguntó a las personas informantes si este gesto permitía expresarle a la otra persona un sentimiento, si permitía demostrar el sentimiento con respecto a lo que se está diciendo o si ninguna de ellas. A esto, seis personas (dos mujeres y cuatro hombres) indican que el gesto les permite demostrar lo que les hace sentir lo que están diciendo, mientras 4 indican que ninguna de estas dos opciones.

Al preguntar a los seis informantes sobre los sentimientos que se expresan por medio de ese gesto, dos señalaron que el gesto permite demostrar sentimientos que generan lo que es dicho (angustia, "el sentimiento que se tiene cuando uno se está frente a un problema"). De las otras cuatro personas, tres indicaron que permite expresarle a la otra persona sentimientos con respecto a lo que está diciendo: preocupación, simpatía o comprensión, camaradería, empatía con la situación del otro; y, una no indicó ningún sentimiento.

De hecho, en la selección múltiple acerca de las funciones del gesto tres personas repitieron la idea de "demostrar lo que hace sentir lo que se está diciendo". En este ítem, se 
repitieron también ideas con respecto a la expresión de sentimientos: una persona seleccionó "demostrarle afecto o desprecio a alguien"; una escribió "para generar angustia o preocupación a la otra persona"; y otra, para "mostrar camaradería, apuro, preocupación, alerta".

De todas las categorías, los adaptadores presentan una especial dificultad para este estudio, por tratarse de movimientos que se realizan de manera poco consciente y sin un objetivo comunicativo desde la perspectiva de Knapp, pero sí poseerlo desde la nuestra. Para esta categoría se realizó un único ítem en el cuestionario: se preguntó a las personas informantes si estarían de acuerdo o en desacuerdo con la idea de que "Este gesto no comunica nada, la gente lo hace porque está nerviosa nada más". Únicamente dos mujeres estuvieron de acuerdo con esta afirmación; una de ellas sí lo utiliza, la otra no. Como vemos la afirmación incluye "nerviosismo", de modo que el gesto igual estaría siendo interpretado como una posible señal que comunique que una persona se encuentra en dicho estado.

A continuación, se realizará un repaso por cada una de las categorías en las que se puede clasificar el ademán según las personas informantes. Primero, si se usara con un significado específico ("iqué torta!") podría ser un emblema; no obstante, para constatar este uso, sería necesario un análisis de una muestra mayor; además, sería necesario establecer en qué grupos el ademán puede tener ese singificado.

Luego, por tratar de comunicar una idea con respecto a lo que se está diciendo o por enfatizarlo (intensificarlo), este gesto sirve como ilustrador. Sin embargo, si expresa ideas con respecto a lo que está diciendo la otra persona (en su turno de palabra), pueden funcionar como regulador (retrocanalizador) por demostrar al otro que se atiende a lo que dice, a la vez que comunica una actitud hacia lo que dice.

También, podría considerarse que funciona como muestra de afecto. Se trata de una categoría problemática, pues según Knapp no se realizan para comunicar, aspecto contrario no solo a la perspectiva que se prefiere en esta pesquisa, sino también a lo que indican las personas informantes. Al respecto, habría que considerar la reformulación de la categoría e incluir que podría tratarse de una manera de expresar cortesía o que busca una menor distancia interpersonal. Esta idea se refuerza con las aclaraciones respecto al contexto de uso, al unir las respuestas de ese ítem con las de otras preguntas sobresale que una posible finalidad de un emisor al utilizar el gesto es expresar solidaridad, compañerismo o un sentimiento de interés hacia la otra persona (hacia lo que está diciendo), a partir de lo cual se puede afirmar que el gesto puede funcionar para expresar cortesía positiva. Finalmente, la idea de que este gesto sea un adaptador se descarta.

\subsection{Función del gesto}

Para establecer la función de gesto, se utilizaron las mismas preguntas que para las categorías, por dar luz con respecto a la finalidad con la que se usa el gesto (para qué sirve). Se preguntó a quienes participaron como informantes por las categorías de repetición y acentuación separadamente, dando ejemplos de cada una de ellas. Estas dos preguntas eran abiertas y permitieron recolectar información para reforzar la categorización del gesto. A continuación, se abarcarán las categorías una a una: sustitución, complementariedad, repetición, regulación, contradicción, y acentuación.

El gesto de agitar la mano puede tener la función de sustitución. Tomando en cuenta los datos de los cinco hablantes que le otorgan un significado traducible en palabras y las dos personas que indican que puede aparecer sin necesidad de hablar al mismo tiempo, se 
comprende que el gesto puede sustituir mensajes verbales, especialmente en su función de emblema con el significado de "iqué torta!”. A partir de las respuestas, también queda claro que una de las funciones del ademán es la complementariedad, especialmente porque permite mostrar al interlocutor las actitudes del hablante con respecto a lo que dice o demuestra la actitud hacia el otro (si se utiliza como retrocanalizador).

Para la función de repetición, solo dos hombres indicaron que puede servir para repetir lo que se está diciendo. Ambos agregaron la aclaración de que lo dicho tendría que ser exclusivamente “qqué torta!”; además, uno incluyó que repetiría también si se utilizan frases como "uy qué mal" o "ahora sí me metió o te metiste en un problema". Las otras ocho personas dijeron que no repetía la información. De ellas, hubo dos personas que indicaron que sí acompaña o complementa lo que se dice (es decir, que es un ilustrador), una indicó que enfatiza (también, ilustrador) y otra indicó que "es muy espontáneo aunque depende de la gravedad de la información”. A pesar de que este gesto no fue mencionado por ninguna persona como utilizado para contradecir, del dato anterior se puede inferir que el gesto también podría funcionar para la contradicción en algún contexto comunicativo específico.

Con respecto a la regulación, se ha señalado hasta aquí que el gesto en estudio puede funcionar como retrocanalizador. Esta es la única utilidad que tiene en la regulación, pues no se puede utilizar -según los hablantes- para iniciar o finalizar una conversación ni para señalar un lugar apropiado para el cambio de turno. Sin embargo, sí es posible que en algunas situaciones sirva para empezar una conversación ("vieras lo que pasó") o finalizarla ("me tengo que ir, tengo que apurarme").

De la acentuación cabe mencionar que durante las diversas preguntas se evidenció que es la función que los hablantes observan con mayor claridad para este gesto. De hecho, las diez personas informantes están de acuerdo con respecto a esta función. Por ejemplo, el gesto sirve para acentuar partes de la historia, de lo cual da cuenta el texto de un informante que describió la situación comunicativa donde usaría el gesto:

\footnotetext{
Me dí cuenta de que un compañero confrontó al profesor por estar dando el curso de manera inadecuada cuando nadie más se había atrevido, uso el gesto al contarle esta situación a otro compañero que faltó a clases el día del suceso
}

Comunica "emoción o entusiasmo acerca de alguna situación, para demostrarla hace se hace [sic] énfasis con este gesto", dice una informante. Otras de las aclaraciones son: "...de hecho lo usaría únicamente para reforzar la importancia de la información que me están diciendo". Lo interesante es que al hacer énfasis también se puede añadir también a lo dicho por el interlocutor: “...si la otra persona le cuenta a uno que se jaló una torta uno responde y hace más énfasis con el gesto”.

\section{Conclusión}

Para concluir, primero es importante retomar el significado del gesto peninsular que es similar al gesto analizado. Como el diccionario y el video traducen el gesto por una única palabra ("mucho"), desde la referencia teórica de este trabajo, el gesto puede ser entendido como emblema, parte de una única categoría. En cambio, en el contexto costarricense el gesto tiene más funciones, como se explica enseguida.

En Costa Rica, el gesto aparece en varias de las categorías y también cumple diversas funciones. Como emblema, puede servir para sustituir, repetir o contradecir lo que se dice. 
Como ilustrador permite enfatizar lo que se está diciendo. Igualmente, como regulador permite contribuir al mantenimiento de la conversación como retrocanalizador (función: regulación). Se debe resaltar que como muestra de afecto permite el establecimiento de relaciones de solidaridad al mostrar camaradería con quien cuenta una historia e, incluso, permitir construir participativamente el sentido de lo que dice un interlocutor (enfatizar en la historia que cuenta quien tiene el turno de palabra).

$\mathrm{Si}$ tomamos en cuenta todas estas categorías y funciones, resulta evidente que muchas de las personas de las familias costarricenses podrían haber estado demostrando al estudiantado su solidaridad y tratando de entablar una relación positiva con la persona aprendiente de español. Estas relaciones son vitales para nuestros alumnos y alumnas, por lo cual es esencial que conozcan sobre este gesto -y otros- para poder interactuar de manera más espontánea y, especialmente, para comprender el mensaje de la comunidad local.

Esta riqueza de funciones y modificaciones del significado posibilitadas por un único gesto recuerda la importancia de incluir la comunicación no verbal en la clase de ELE. Especialmente, evidencia la necesidad de enseñar sobre los gestos para lograr que las estudiantes y los estudiantes puedan comunicarse efectivamente en la nueva cultura.

Este trabajo es un insumo para abordar el gesto en nuestras clases. No obstante, el conocimiento de un único comportamiento kinésico no es suficiente para abordar el tema de la comunicación no verbal kinésica del modo que se requiere en el aula. Por ello cabe afirmar que en Costa Rica hace falta realizar más trabajos que permitan al profesorado de ELE dar una respuesta a las preguntas de sus estudiantes; se recomienda abarcar otros gestos y compararlos con los materiales disponibles en línea o en otros formatos sobre gestos en el español en general.

Finalmente, no debe olvidarse que los gestos no tienen un significado último. Al abordar este gesto o cualquier otro en clase, se debe explicar a las estudiantes y los estudiantes que las posibles funciones o interpretaciones para el gesto no significa enseñarles "la verdad última sobre lenguaje corporal", como quieren hacer algunos manuales disponibles en el mercado que solo sirven para confundir a su público meta en general. Las personas aprendices de ELE requieren los insumos necesarios para interpretar la función del gesto en el contexto en que este es utilizado.

\section{Bibliografía}

Betti, S. y Costa, V. (2006). Para una didáctica contrastiva del lenguaje gestual. XVII Congreso Internacional de la ASELE: Las destrezas orales en la enseñanza del español L2-LE. http://cvc.cervantes.es/ensenanza/biblioteca_ele/asele/pdf/17/17_0365.pdf [Consulta 11 de diciembre del 2014].

Bravo, D. (2012). 4 Cortesía lingüística y comunicativa. Por S. los Heros y M. Niño-Murcia, (Eds.). Fundamentos y modelos del estudio pragmático y sociopragmático del español. Georgetown University Press.

Bolaños-Carpio, A. (2009). La risa aislada. Káñina. 33 (2), 151-162. http://www.latindex.ucr. ac.cr/kan014/kan014-09.pdf [Consulta 24 de octubre de 2013].

Ciarra-Tejada, A. (2011). El lenguaje no verbal: los diccionarios de gestos disponibles en la red como recurso de enseñanza/aprendizaje en ele, propuesta didáctica para el 
nivel intermedio (B1-B2). XXII Congreso Internacional de la ASELE. La Red y sus aplicaciones en la enseñanza-aprendizaje del español como lengua extranjera. http:// cvc.cervantes.es/ensenanza/biblioteca_ele/asele/pdf/22/22_0017.pdf [Consulta 10 de diciembre de 2014].

Gaviño-Rodríguez, V. (Dir. y Coord.). (2012). Diccionario de gestos españoles. Diccionario de español coloquial. http://www.coloquial.es/es/diccionario-de-gestos-espanoles/ [Consulta 20 de octubre del 2013].

Iruela, A. (s.f.). Sin palabras. Nivel B1. Video ELE. http://www.videoele.com/B1_Sin-palabras. html [Consulta 10 de diciembre de 2014].

Knapp, M. (1982). La comunicación no verbal: El cuerpo y el entorno. Barcelona: Paidós.

Martinell-Gifre, E. y Ueda, H. (Eds). (s.f.). Mucho. Diccionario de gestos españoles. http:// lecture.ecc.u-tokyo.ac.jp/ cueda/gakusyu/gestos/arc-center.php?key=37a [Consulta 11 de diciembre de 2014].

Monterrubbianesi, M. (2013). La comunicación no verbal en el aula: una propuesta de clasificación y análisis de los gestos para las clases de E/LE. marcoELE Revista de didáctica ELE. 16. http://marcoele.com/descargas/16/monterubbianesi-comunicacion_ no_verbal.pdf [Consulta 10 de diciembre de 2014].

Payrató, L. (2006). Discurso oral y multimodalidad: aspectos introductorios. ORALIA. 9, 259275.

Poyatos, F. (2002). Nonverbal Communication across Disciplines. Volume 2: paralanguage, kinesics, silence, personal and environmental interaccion. Philadelphia: John Benjamins Publishing Company.

Sánchez-Benítez, G. (2009). La comunicación no verbal. marcoELE, 8. http://marcoele.com/ descargas/china/g.sanchez_comunicacionnoverbal.pdf [Consulta 11 de diciembre de 2014].

Yagüe-Barredo, A. (2004). ELAO y ELE. Hablando por los codos. redELE: Revista Electrónica de Didáctica ELE. 1. http://www.mecd.gob.es/dctm/redele/Material-RedEle/ Revista/2004_01/2004_redELE_1_15Yague.pdf?documentId=0901e72b80e06d00 [Consulta10 de diciembre de 2014]. 